Research Paper

\title{
Sequential Therapy with Gemcitabine and Carboplatin Followed by Paclitaxel as First Line Treatment for Advanced Urothelial Cancer
}

\author{
Joseph G Kattan ${ }^{1 凶}$, Celine Y Boutros ${ }^{1}$, Fadi S Farhat ${ }^{1}$, Georges Y Chahine ${ }^{1}$, Khaled M Musallam¹, \\ Marwan G Ghosn
}

1. Cancer Research Group (CRG) Collaborative Group, Hotel-Dieu de France University Hospital;

2. American University of Beirut Medical Center, Beirut, Lebanon.

$\square$ Corresponding author: Joseph G. Kattan, MD. Ass. Prof. Of Hematology-Oncology, Department of Hematology-Oncology, Hôtel-Dieu de France University Hospital, Achrafieh, Blvd Alfred Naccache, Beirut, Lebanon. Tel: 00961-3-63 59 13, Fax: 00961-1-42 49 42, E-mail: jkattan62@hotmail.com.

() Ivyspring International Publisher. This is an open-access article distributed under the terms of the Creative Commons License (http://creativecommons.org/ licenses/by-nc-nd/3.0/). Reproduction is permitted for personal, noncommercial use, provided that the article is in whole, unmodified, and properly cited.

Received: 2012.02.10; Accepted: 2012.08.31; Published: 2012.09.06

\begin{abstract}
Objective: Gemcitabine and platinum-based compounds represent the new standard combination therapy for bladder cancer. In this study, we evaluate the efficacy and safety of gemcitabine and carboplatin followed sequentially by paclitaxel in 27 patients with advanced transitional cell carcinoma.

Methods: This phase II multicentre study was based on the doublet gemcitabine $800 \mathrm{mg} / \mathrm{m} 2$ and carboplatin area under the concentration-time curve 2 on days I and 8 every 21 days for 4 cycles, followed sequentially by paclitaxel $60 \mathrm{mg} / \mathrm{m}^{2} / \mathrm{w}$ for 12 consecutive weeks. The disease was assessed after each sequence.

Results: Primary tumor was localized in the bladder and renal pelvis in 25 and 2 patients, respectively. Twenty patients completed all 4 cycles of the gemcitabine and carboplatin sequence. Mean number of cycles was 3.5 (range I to 4). Toxicities were mainly hematologic, including Grade 3 neutropenia and anemia in 3 patients. Objective response was noted in II pts (40.7\%), including I complete response (CR) and I0 partial responses (PR). Three patients had stable disease and II progressed. Among the 20 patients, 14 received the second sequence. Mean number of paclitaxel injections was 7 (range 2 to 12). Toxicities were limited to diarrhea and neurotoxicity in I patient each. Objective response was documented in 6 patients (30\%) (3 CR and 3 PR), including the improvement of PR into CR in 2 patients. Median duration of response was 6 months. After a median follow-up of 7 months, 21 patients died and 6 remained alive, including 2 who maintained CR and I PR.

Sixteen patients had locally advanced disease and II had metastatic disease, better prognostic was noticed with patients with locally advanced disease.

Conclusion: the sequential approach of treatment for advanced urothelial cancer using gemcitabine and carboplatine followed by paclitaxel seems to be a safer alternative to the combined triplet, but due to the limited number of patients this study failed to improve outcome. Further investigations with larger population are required.
\end{abstract}

Key words: bladder cancer, gemcitabine, carboplatin, paclitaxel, sequential therapy.

\section{INTRODUCTION}

Bladder cancer (BC) is the fourth most common cancer in men, with an estimated of 70,980 new cases diagnosed and 14,330 deaths in the US in 2009.1 Transitional cell carcinoma (TCC) remains the most com- 
mon histological subtype. In the 1990s, the methotrexate, vinblastine, doxorubicin and cisplatin (MVAC) regimen was considered the gold-standard of chemotherapy for the treatment of patients with advanced or metastatic TCC. ${ }^{2-4}$ However, the MVAC regimen was relatively toxic and the search for a safer alternative ensued. Several newer agents including paclitaxel, docetaxel and gemcitabine have demonstrated substantial single-agent activity in the treatment of advanced TCC. ${ }^{5-7}$ Phase II trials combining these agents with platinum-based compounds or with each other as doublets have also shown favorable activity. ${ }^{8-11}$ In a randomized phase III study, the combination of gemcitabine and cisplatin had similar efficacy to the MVAC regimen with lower toxicity, thus becoming the new gold-standard treatment for advanced TCC. ${ }^{12}$ Other attempts to improve outcome included combining paclitaxel to platinum and gemcitabine regimens, as triplet therapy. However, the simultaneous administration of the three drugs increased toxicity without evidence of improved efficacy. ${ }^{13}$ In this study, we evaluate the activity and safety of gemcitabine and carboplatin followed sequentially by paclitaxel in patients with advanced TCC.

\section{PATIENTS AND METHODS}

\section{Eligibility criteria}

Patients with histologically confirmed locally advanced or metastatic TCC of the urothelial tract (bladder, ureter, or renal pelvis), were enrolled in 3 different medical centres from September 2004 till September 2007 that was either locally advanced or metastatic. All patients were required to have at least one measurable lesion. No prior chemotherapy was allowed, unless in adjuvant or neoadjuvant setting with more than one year chemotherapy-free interval since the last dose. Previous intravesical treatment was allowed if the most recent intravesical therapy completed was more than one month prior to study enrolment. Prior irradiation to the bladder was not allowed. Additional inclusion criteria included: age between 18 and 75 years, Eastern Cooperative Group Performance Status (ECOG PS) $\leq 2$. Hematologic variables: hemoglobin $\geq 10 \mathrm{~g} / \mathrm{dl}$, leukocyte count $\geq 3000$ $/ \mathrm{mm}^{3}$, platelet count $\geq 100000 / \mathrm{mm}^{3}$; creatinine clearance $\geq 30 \mathrm{ml} / \mathrm{min}$. Hepatic function variables: total serum bilirubin $\leq 1.5$ upper limit of normal (ULN), alanine aminotransferase (ALT) and aspartate aminotransferase (AST) $\leq 2.5 \mathrm{ULN}(\leq 3.5 \mathrm{ULN}$ in case of liver metastasis).

Patients were excluded if they had a history of other malignancy (except non-melanoma skin cancer), active cardiac disease necessitating continuous therapy, or evidence of neurological disturbance or cerebral metastasis.

The study protocol was approved by the institutional review board at each center and written informed consent was obtained from each patient prior to enrolment.

\section{Baseline evaluations}

Baseline evaluation included a medical history and physical examination; complete blood count $(\mathrm{CBC})$ and differential; serum electrolyte, liver enzymes, bilirubin, and creatinine levels; urinalysis; chest radiography; and electrocardiography. All patients underwent baseline crosssectional imaging including a computed tomography (CT) scan or magnetic resonance imaging scan of the abdomen and pelvis. If clinically indicated chest CT scan and bone scan were performed.

\section{Treatment schedule}

Patients received treatment through the following regimen: intravenous (IV) gemcitabine $800 \mathrm{mg} / \mathrm{m}^{2}$ and IV carboplatin area under the concentration-time curve 2, on Days 1 and 8. The regimen was repeated every 21 days for 4 cycles, followed by IV paclitaxel 60 $\mathrm{mg} / \mathrm{m}^{2}$ weekly for 12 weeks. Patients received standard antiemetic prophylactic and paclitaxel premedication.

\section{Toxicity assessment and dose modification}

Toxicity assessment was performed on days 1 and 8 of each cycle using the Common Toxicity Criteria (CTC) of the National Cancer Institute version 3.0. All patients received the full dose of gemcitabine and carboplatin on Day 1 of the first cycle.

On Day 1 of each cycle, full doses of both agents were administered if leukocyte count was $\leq$ grade 1 and the platelet count was $\geq 100000 / \mathrm{mm}^{3}$. If the leukocyte count was grade 2or the platelet count was $75000-100000 / \mathrm{mm}^{3}, 75 \%$ of the doses of both drugs were administered. If the leukocyte count was $\geq$ grade 3 or the platelet count was $\geq$ grade 2 , then treatment was delayed for one week, and reinitiated at $75 \%$ doses of both agents when blood counts improved to baseline conditions. If discontinuation is required for more than 2 weeks, the patient was discontinued from the study.

For Day 8 , both agents were administered if the leukocyte count was $\leq$ grade 2 and the platelet count was $\leq$ grade 1 . If not, treatment on day 8 was cancelled.

Paclitaxel was delayed for one week, if the leukocyte count was $\geq$ grade 3 or the platelet count was $\geq$ 
grade 2 . If discontinuation is required for more than 1 week, the patient was discontinued from the study.

In the event of neutropenic fever, subsequent doses of all drugs were reduced by $25 \%$. Patients with reversible Grade 3 or 4 nonhematologic toxicity (with the exception of nausea, emesis, or alopecia) had doses of the offending agent or agents withheld until the toxicity resolved to $\leq$ Grade 1 . Treatment was resumed using $75 \%$ of the doses of the offending agents. Patients who developed irreversible Grade 3 or 4 nonhematologic toxicity or who had Grade 3 or 4 neurotoxicity were removed from the protocol.

All patients who received at least one dose of treatment were included in the toxicity analysis.

\section{Response assessment}

Tumor response assessment was performed after completion of gemcitabine plus carboplatin sequence and then after completion of paclitaxel. Reevaluation included a repeat of all previously abnormal radiologic studies with repeat of objective tumor measurements. Treatment efficacy was evaluated using the Response Evaluation Criteria In Solid Tumors (RECIST).

Complete response (CR) was defined as the disappearance of all target lesions. Partial response (PR) was defined as $30 \%$ decrease in the sum of the longest diameter of target lesions.

Progressive disease (PD) was defined as $20 \%$ increase in the sum of the longest diameter of target lesions. Stable disease (SD) was defined as small changes that did not meet above criteria.

To ensure comparability baseline imaging studies and subsequent follow up studies used to assess response were performed using identical techniques throughout the study.

\section{Statistical analysis}

The current trial was designed to detect a response rate of $40 \%$ as compared to a minimal, clinically meaningful response rate of $20 \%$. The primary end point of this study was to assess the efficacy of the sequence regimen as first-line treatment in advanced uorthelial cancer, measured as an objective response rate (ORR). Secondary end points included overall survival (OS), duration of response, time to progression (TTP) and toxicity. Survival time was defined as the time from registration to time of last follow up or death due to any cause. Survival curves were calculated according to the methods of Kaplan and Meier.

TTP was defined as the time from registration to documentation of disease progression. If a patient died without documentation of disease progression, the patient was considered to have had tumour pro- gression at the time of their death unless there was sufficient documented evidence to conclude that no progression occurred prior to death.

Fleming's single-stage design was applied for the sample size determination and showed that the hypothesis $\mathrm{Ha}$ should be rejected if the number of responses $\leq 9$ and the hypothesis $\mathrm{H} 0$ should be rejected if the number of responses $>9$, with a sample size of 25. The statistical data were obtained using an SPSS software version 16.0 (SPSS, Chicago, IL, USA).

\section{RESULTS}

\section{Patient characteristics}

A total of 27 patients were enrolled in this study. The mean age was 68 years (range $44-79$ years) with a male to female ratio of 22:5. Primary tumor was localized in the bladder $(n=25)$ and in the renal pelvis $(n=2)$. Sixteen patients had locally advanced disease and 11 had metastatic disease at the time of diagnosis. Metastasis sites included: lung $(n=5)$, liver $(n=3)$, bone $(n=4)$ and adrenal gland $(n=1)$. Twelve patients had undergone previous radical cystectomy and 1 patient had received intravesical treatment. Patients were classified following the Bajorin prognostic factor ${ }^{18}$.

Table I. Patient Characteristics.

$\begin{array}{llc}\text { Characteristic } & \text { Group } & \begin{array}{c}\text { Percentage } \\ \text { (number) }\end{array} \\ \text { Sex } & \text { Male } & 81.5 \%(22) \\ & \text { Female } & 18.5 \%(5) \\ & & \\ \text { Bajorin prognostic factor } & 0 & 74 \%(20) \\ & 1 & 26 \%(7) \\ & 2 & 0 \%(0) \\ \text { Primary tumor location } & & \\ & \text { bladder } & 92.5 \%(22) \\ & \text { renal pelvis } & 7.5 \%(5) \\ & & \\ \text { Disease } & & \\ & & \\ & & \\ & & \\ & \text { Locally advanced } & \end{array}$

\section{Dose administration}

Twenty patients completed all 4 cycles of gemcitabine and carboplatin. The mean number of completed cycles was 3.5 (range, 1-4). Seven patients stopped the treatment at an earlier stage of the study 
because of disease progression $(n=6)$ or personal request $(n=1)$. Dose-reductions of $25 \%$ were required in 2 of 27 patients for hematologic toxicities.

Among the 20 patients who completed the first sequence, 6 patients could not receive the sequence paclitaxel for loss of follow-up $(\mathrm{n}=3)$ or personal request $(\mathrm{n}=3)$. In the remaining 14 patients, the mean number of paclitaxel injections was 7 (range 2-12). Dose-reductions of $25 \%$ were required in 2 patients due to myelosuppression.

\section{Efficacy}

Based on an intent-to-treat analysis, at the completion of gemcitabine plus carboplatin sequence, there were $1 \mathrm{CR}(3.7 \%)$ and $10 \mathrm{PR}(37.0 \%)$ giving an ORR of $40.7 \%$. Responses were achieved in 8 out of 16 patients with either a primary unresectable tumor or metastases limited to the lymph nodes, and in 3 out of 11 patients with distant metastases. Three patients had SD and 11 had PD.

At the completion of the paclitaxel sequence, there were 3 CR $(15 \%)$ and 3 PR $(15 \%)$ giving an ORR of $30 \%$. Responses were achieved in patients who already responded to the first sequence (gemcitabine plus carboplatin) including 2 PR who were rendered $\mathrm{CR}$ after paclitaxel sequence. All responders were patients with either primary unresectable tumor or metastases confined to lymph nodes. Four patients had SD and 4 had PD. Of note, 7 of the patients who achieved PR or had SD after the first sequence $(n=13)$ progressed after the second sequence.

The median duration of response was 6 months and the median TTP was 5 months (Figure 1A). After a median follow-up of 7 months, 21 patients died and 6 remained alive, including 2 who maintained $\mathrm{CR}$ and one who maintained PR. The median OS was 7 months (Figure 1B).

\section{Toxicity}

Toxicities experienced during the study are summarized in Table 2. Myelosuppression was the most common toxicity during gemcitabine plus carboplatin treatment. Two patients experienced Grade 3 neutropenia and one patient required hospitalization for the treatment of febrile neutropenia. Grade 3-4 thrombocytopenia occurred in 2 patients, yet there were no bleeding episodes reported. Three patients experienced Grade 3 anemia and 11 patients required red blood cell transfusions. Nonhematologic toxicity was uncommon. One patient experienced a Grade 2 hypersensitivity reaction and one patient had Grade 3 nausea/vomiting.

In the paclitaxel sequence, there were no episodes of Grade 3-4 neutropenia or thrombocytopenia. One patient experienced Grade 3 anemia and 3 patients required red blood cell transfusions. Nonhematologic toxicities included Grade 2 neuropathy in one patient and Grade 3 diarrhea in another patient.

Table 2. Observed toxicities during the course of treatment.

\begin{tabular}{lll}
\hline Toxicity & $\begin{array}{l}\text { Gemcitabine/Carboplatin } \\
\text { sequence }\end{array}$ & $\begin{array}{l}\text { Paclitaxel } \\
\text { sequence }\end{array}$ \\
\hline Hematologic & & \\
Neutropenia & 8 & 5 \\
$\quad$ Grade 1-2 & 2 & 0 \\
$\quad$ Grade 3 & 0 & 0 \\
$\quad$ Grade 4 & 4 & 4 \\
Thrombocytopenia & 1 & 0 \\
$\quad$ Grade 1-2 & 1 & 0 \\
$\quad$ Grade 3 & & \\
Grade 4 & 16 & 8 \\
Anemia & 3 & 1 \\
$\quad$ Grade 1-2 & 0 & 0 \\
$\quad$ Grade 3 & & 0 \\
$\quad$ Grade 4 & 1 & 0 \\
Myelosupression-related complications & 1 & 0 \\
$\quad$ Febrile neutropenia & 0 & 3 \\
Platelets transfusion & 11 & \\
$\quad$ Bleeding episodes & & 0 \\
$\quad$ Red blood cells transfusion & 1 & 1 \\
Nonhematologic (Grade 3-4) & 0 & \\
Nausea/vomiting & & \\
Diarrhea & &
\end{tabular}



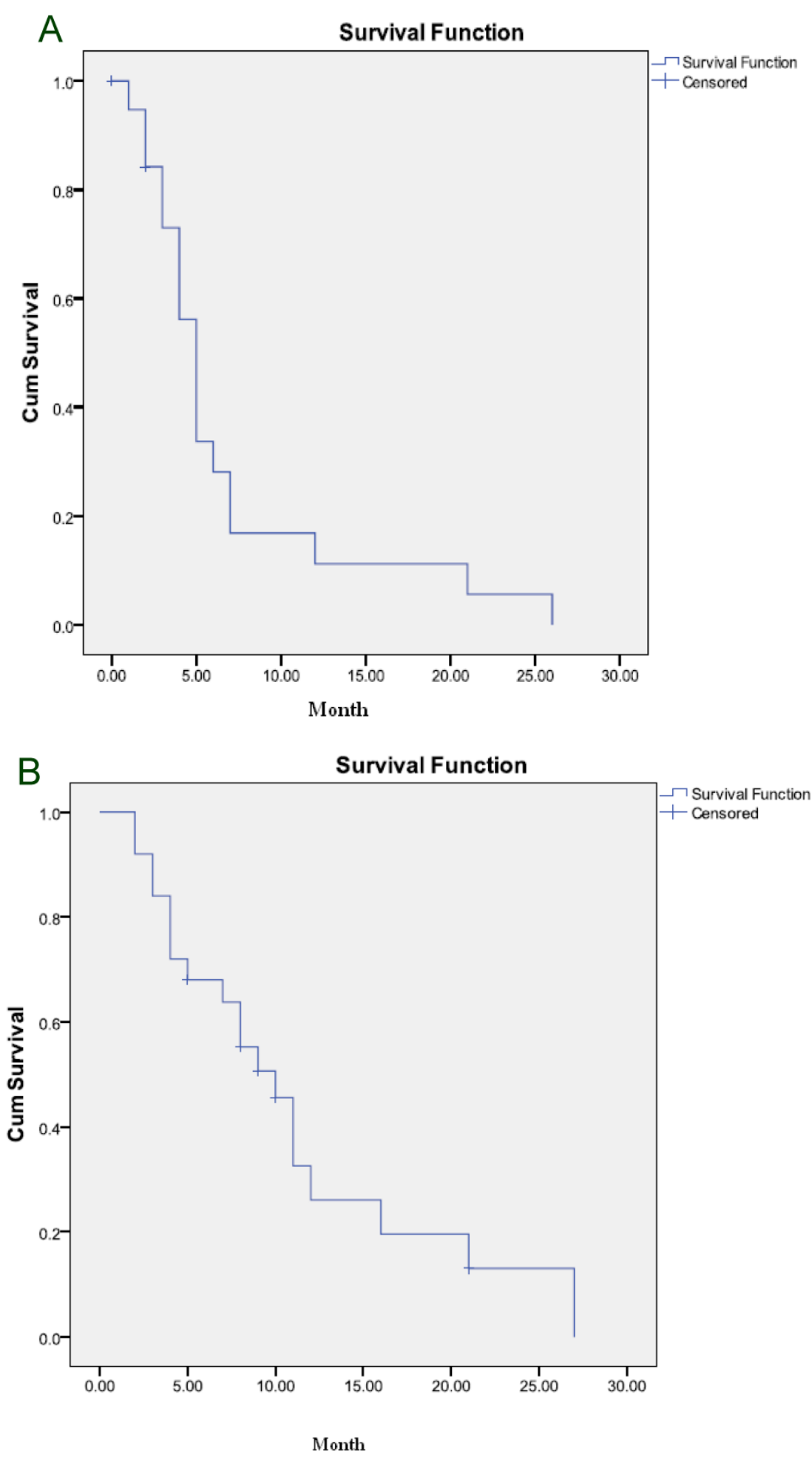

Figure I. (A) Time to progression to the whole group. (B) Overall survival for the whole group.

\section{DISCUSSION}

Despite the unprecedented activity of the MVAC regimen in advanced urothelial cancer, its limitations were readily apparent as a result of the treatment related toxicities ${ }^{2}$. Response rates up to $72 \%$ in first-line therapy were reported 2-4; however, toxicity was significant with a high incidence of drug-related deaths, nadir sepsis, and Grade 3+ myelosuppression (up to $58 \%) .^{2}$ 
Core and long-term results of phase III trials redefined the treatment approach by showing that the combination of gemcitabine and cisplatin is equivalent to MVAC (ORR $49 \%$ vs. $46 \%$, respectively) with lower incidence of toxicity (Grade 3-4 neutropenia $82 \%$ vs. $71 \%$, respectively; neutropenic fever $14 \%$ vs. $2 \%$, respectively; neutropenic sepsis $12 \%$ vs. $1 \%$, respectively). ${ }^{12,14}$

The search for a more ideal regimen yet continued. Carboplatin has been substituted for cisplatin in various combinations to decrease nephrotoxicity. ${ }^{15}$ Moreover, the individual dosage of each of our drug protocol is rather at the lowest limit with respect to the renal function fragility of these patients, as currently used by Vandenput et al. and Rozzi et al. in treating gynecologic and lung tumors. ${ }^{19-20}$ In parallel to this work, several studies showed that paclitaxel as monotherapy is an active and safe regimen in advanced urothelial cancer patients. ${ }^{5}$ However, its addition to the gemcitabine and platinum regimens increased toxicity without evidence of improved treatment efficacy. ${ }^{13,16,17}$

In this trial, rather than simultaneously adding paclitaxel to gemcitabine and carboplatin, we attempted to improve treatment efficacy and safety using a sequential therapy with gemcitabine and carboplatin followed by paclitaxel. The ORR rate of $30 \%$ based on intent-to-treat analysis seems slightly lower than results of previous regimens combining gemcitabine plus carboplatin, essentially because of the early withdrawal of 7 patients. ${ }^{11-14}$ Moreover, 11 patients were excluded and did not receive paclitaxel sequence. Responses were mainly achieved in advanced unresectable urothelial carcinoma with positive lymph nodes, and without evidence of visceral metastases.

It is interesting to note that this regimen was relatively well tolerated. Fourteen of 27 patients (51\%) received $100 \%$ of the regimen and few patients required dose reduction. Grade 3 and 4 neutropenia and febrile neutropenia were infrequent and substantially lower than rates reported for the MVAC, gemcitabine plus carboplatin regimen, and triple drug regimen of gemcitabine, carboplatin and paclitaxel.2,12-17 Grade 3 and 4 nonhematologic toxicities were also rare. These results demonstrate the sequential regimen is safe in this population of patients.

Several limitations exist in this study. First of all, recruitment was very slow, since it took 3 years to recruit 27 patients. Also, the follow-up duration was relatively short, with a median of 7 months. A definitive survival benefit has not been shown in this trial, since the median OS was 7 months only which may be attributed to the small number of patients included. In addition, some patients who achieved PR or had SD after the gemcitabine plus carboplatin sequence showed disease progression under paclitaxel treatment. These results indicate that, although the monotherapy in the second sequence of the regimen may be active in some patients by enhancing PR into CR, it seems to be insufficient in others and unable to circumvent rapid disease progression, especially in patients with visceral metastases. This could reflect the aggressive natural history of this disease in these patients, since delayed paclitaxel could not circumvent tumor aggressiveness.

Our study demonstrates that a sequential regimen of gemcitabine and carboplatin followed by paclitaxel is highly tolerable when compared to simultaneous administration of the three drugs and could offer a smooth alternative for advanced urothelial cancer chemotherapy. However, larger studies with a larger number of patients are warranted to better define optimal dosing and schedule of this regimen before concluding that efficacy is lower with sequential regimen.

\section{CONFLICT OF INTEREST}

The authors have declared that no conflict of interest exists.

\section{REFERENCES}

1. Jemal A, Siegel R, Ward E, et al. Cancer statistics, 2009. CA Cancer J Clin 2009;59: 225-49.

2. Sternberg CN, Yagoda A, Scher HI, et al. Methotrexate, vinblastine, doxorubicin, and cisplatin for advanced transitional cell carcinoma of the urothelium. Cancer 1989;64: 2448-58.

3. Logothetis CJ, Dexeus FH, Finn L, et al. A prospective randomized trial comparing MVAC and CISCA chemotherapy for patients with metastatic urothelial tumors. J Clin Oncol 1990;8: 1050-5.

4. Loehrer P, Einhorn LH, Elson PJ, et al. A randomized comparison of cisplatin alone or in combination with methotrexate, vinblastine and doxorubicin in patients with metastatic urothelial carcinoma of the bladder: a cooperative group study. J Clin Oncol 1992;10: 1066-73.

5. Roth B, Dreicer R, Einhorn LH, et al. Significant activity of paclitaxel in advanced transitional-cell carcinoma of the urothelium: a phase II trial of the Eastern Cooperative Oncology Group. J Clin Oncol 1994;12: 2264-70.

6. McCaffrey JA, Hilton S, Mazumdar M, et al. Phase II trial of docetaxel in patients with advanced or metastatic transitional-cell carcinoma. J Clin Oncol 1997;15: 1853-7.

7. Stadler WM, Kuzer T, Roth B, et al. A phase II trial of single gemcitabine in previously untreated patients with metastatic urothelial cancer. J Clin Oncol 1997;15: 3394-8.

8. Gitliz BJ, Baker C, Chapman Y, et al. A phase II study of gemcitabine and docetaxel therapy in patients with advanced urothelial carcinoma. Cancer 2003;98: 1863-9.

9. Vaughn DJ, Manola J, Dreicer R, et al. Phase II study of paclitaxel plus carboplatin in patients with advanced carcinoma of the urothelium and renal dysfunction. A trial of the Eastern Cooperative Oncology Group. Cancer 2002;95: 1022-7.

10. Meluch AA, Greco FA, Burris HA, et al. Paclitaxel and gemcitabine chemotherapy for advanced transitional-cell carcinoma of the urothelial tract: a phase II of the Minnie Pearl Cancer Research Network. J Clin Oncol 2001; 19: 3018-24. 
11. Nogue-Aliguer M, Carles J, Arrivi A, et al. Gemcitabine and carboplatin in advanced transitional cell carcinoma of the urothelial tract. An alternative therapy. Cancer 2003;97: 2180-6.

12. Van Der Maase H, Hansen SW, Roberts JT, et al. Gemcitabine and cisplatin versus methotrexate, vinblastine, doxorubicin, and cisplatin in advanced or metastatic bladder cancer: results of a large, randomized, multinational, multicenter, phase III study. J Clin Oncol 2000;18: 3068-77.

13. Hainsworth JD, Meluch AA, Litchy S, et al. Paclitaxel, carboplatin, and gemcitabine in the treatment of patients with advanced transitional cell carcinoma of the urothelium: a phase II trial of the Minnie Pearl Cancer Research Network. Cancer 2005;11: 2298-303.

14. Von der Maase H, Sengelov L, Roberts JT, et al. Long-term survival results of a randomized trial comparing gemcitabine plus cisplatin with methotrexate, vinblastine, doxorubicine, plus cisplatin in patients with bladder cancer. J Clin Oncol 2005;23: 4602-8.

15. Bellmunt J, Ribas A, Eres N, et al. Carboplatin-based versus cisplatin-based chemotherapy in the treatment of surgically incurable advanced bladder carcinoma. Cancer 1997;80: 1966-72.

16. Hussain M, Vaishampayan V, DU W, et al. Combination paclitaxel, carboplatin and gemcitabine is an active treatment for advanced urothelial cancer. J Clin Oncol 2001;19: 2527-33.

17. Bellmunt J, Von der Maase H, Mead GM, et al. Randomized phase III study comparing paclitaxel/cisplatin/gemcitabine (PCG) and gemcitabine/cisplatin (GC) in patients with locally advanced (LA) or metastatic $(\mathrm{M})$ urothelial cancer without prior systemic therapy; EORTC30987/Intergroup Study. J Clin Oncol. 2007;25(18S). ASCO Annual Meeting Proceedings Part I.

18. Bajorin DF, Dodd PM, Mazumdar M, et al. Long-term survival in metastatic transitional-cell carcinoma and prognostic factors predicting outcome of therapy. J Clin Oncol. 1999 Oct;17(10):3173-81.

19. Vandenput I, Vergote I, Neven $P$, et al. Weekly paclitaxel-carboplatin regimen in patients with primary advanced or recurrent endometrial carcinoma. Int J Gynecol Cancer. 2012 May;22(4):617-22.

20. Rozzi A, Nardoni C, Corona M, et al. Weekly regimen of paclitaxel and carboplatin as first-line chemotherapy in elderly patients with stage IIIB-IV non small cell lung cancer (NSCLC): results of a phase II study. J Chemother. 2010 Dec;22(6):419-23. 